\title{
Impacto en la calidad de vida por la asociación entre trastornos de sueño y síndrome de intestino irritable
}

\section{Impact on the Quality of Life of the Association between Sleep Disorders and Irritable Bowel Syndrome}

Edward A. Cáceres Méndez, MD, MSc, ${ }^{1}$ Andrés J. Gómez, MD, ${ }^{2}$ Alberto Rodríguez Varón, MD, ${ }^{3}$ Jaime Alvarado Bestene, MD, ${ }^{3}$

Patricia Hidalgo, MD, ${ }^{4}$ Mauricio Medina. ${ }^{5}$

1 Residente de tercer año de medicina interna, Pontificia Universidad Javeriana. Bogotá, Colombia.

2 Especialista en medicina interna, Pontificia

Universidad Javeriana. Bogotá, Colombia.

3 Profesor titular de medicina interna y

gastroenterología, Pontificia Universidad Javeriana,

Hospital Universitario San Ignacio. Bogotá,

Colombia.

4 Profesora asociada de medicina interna y neumología, Pontificia Universidad Javeriana, Hospital Universitario San Ignacio. Bogotá, Colombia.

5 Estudiante de medicina - semillero AOS, Pontificia Universidad Javeriana. Bogotá, Colombia.

Fecha recibido: $27-08-15$

Fecha aceptado: 26-01-16

\section{Resumen}

Objetivos: determinar si la presencia de alteraciones del sueño se asocia con el deterioro en la calidad de vida, medida a través del cuestionario SF-36, en pacientes con síndrome de intestino irritable (SII).

Métodos: se diseñó un estudio de corte transversal en el que se incluyeron individuos con SIl seleccionados con los criterios de Roma III, a los que se les aplicó la escala de Epworth, el cuestionario de Pittsburgh y los criterios para síndrome de piernas inquietas (SPI); de manera simultánea, se les realizó la evaluación de calidad de vida usando la escala SF-36.

Resultados: se incluyeron 80 pacientes con SII, la mayoría mujeres; el $81 \%$ presentó algún tipo de trastorno del sueño determinado por la alteración en 1 o más escalas. Al estratificar los pacientes con y sin trastornos del sueño, se observó que el subgrupo con trastorno del sueño se asoció con mayores alteraciones en la calidad de vida, con OR 4,8125, IC 95\%: 1,17-19,02, p < 0,0076, diferencia estadísticamente significativa.

Conclusiones: en este estudio se encontró que hasta un $81 \%$ de los pacientes con SII presentan trastornos de sueño, y que las alteraciones del sueño en pacientes con SII se asocian con un mayor compromiso de la calidad de vida según la escala SF-36.

\section{Palabras clave}

Síndrome de intestino irritable, trastornos del sueño, calidad de vida, SF-36.

\section{Abstract}

Objectives: The objectives of this study were to determine if sleep disorders in patients with irritable bowel syndrome (IBS) were associated with impaired quality of life as measured by the SF36 questionnaire.

Methods: This is cross-sectional study in which individuals with IBS according to the Rome III criteria were evaluated for sleep disorders with the Epworth Sleepiness Scale questionnaire and the Pittsburgh Sleep Quality Index (PSQI) questionnaire. They were also evaluated for restless legs syndrome (RLS) and, simultaneously, the Short Form (36) Health Survey was used to evaluate participants' quality of life.

Results: Eighty patients with IBS, mostly women, were included in this study. $81 \%$ had some type of specific sleep disorder as measured by at least one of the questionnaires. Patients were groups into those who had sleep disorders, and those who did not. The group which had sleep disorders had statistically significantly more alterations in quality of life (OR $4.8125,95 \% \mathrm{Cl}: 1.17$ to $19.02, \mathrm{p}<0.0076)$.

Conclusions: This study found that up to $81 \%$ of IBS patients have sleep disorders and that sleep disturbances in patients with IBS are associated with decreased quality of life according to the SF36 scale.

Keywords

Irritable bowel syndrome, sleep disorders, quality of life, SF36. 


\section{INTRODUCCIÓN}

El síndrome de intestino irritable (SII) es un trastorno gastrointestinal funcional muy frecuente y que genera un alto costo para los sistemas de salud; en países como Estados Unidos alcanza los 20 billones de dólares, derivado del mayor consumo de servicios de salud (1).

Su síntoma principal es el dolor abdominal crónico o recurrente, al igual que la alteración en el hábito intestinal. El diagnóstico actual de dicha entidad se realiza basado en los criterios de Roma III establecidos en 2006 (2). Con frecuencia, se encuentra una asociación del SII con síndromes y síntomas que comprometen otros sistemas, entre los que se destacan cefalea ( $34 \%$ a $50 \%$ de los pacientes), fibromialgia $(32,5 \%)$, dolor lumbar (30\%), fatiga crónica $(51 \%)$, dispareunia $(52,3 \%)$ y cambios en la frecuencia urinaria (50\%) (3-5). Se cree que la asociación con estos trastornos repercute en la calidad de vida de los individuos, observando una mayor limitación en el desempeño de sus actividades diarias $(3,6)$.

En los últimos años se ha dado importancia a la asociación entre SII y otras alteraciones como los trastornos de sueño, y se ha buscado establecer si estas asociaciones pueden condicionar un mayor deterioro de la calidad de vida $y$, eventualmente, ser causa de refractariedad o pobre respuesta al manejo del SII $(5,7,8)$. Al tener en cuenta la prevalencia del SII, los esfuerzos actuales que se están realizando para validar e implementar el estudio de calidad de vida en pacientes con SII y la posible asociación entre SII y trastornos de sueño se considera pertinente realizar un estudio para determinar la frecuencia de alteraciones de sueño en pacientes con SII y el impacto en la calidad de vida cuando se presenta esta asociación.

\section{MATERIALES Y MÉTODOS}

Con el fin de determinar la frecuencia de las alteraciones de sueño en un grupo de pacientes con SII y de determinar si la asociación entre los 2 trastornos altera aún más la calidad de vida de los pacientes, se diseñó un estudio observacional descriptivo de corte transversal en el que se incluyeron pacientes mayores de 18 años y menores de 65 años con SII definido por los criterios de Roma III, a quienes se les realizó la escala de somnolencia de Epworth validada para Colombia (la cual establece somnolencia diurna si el puntaje es mayor a 10), el cuestionario de Pittsburgh para calidad de sueño (clasifica como mal dormidor a los pacientes con puntaje mayor a 5) y los criterios para piernas inquietas (siendo positivo el paciente con 2 de 4 criterios). A todos se les aplicó la escala SF-36 para determinar la calidad de vida en pacientes con síndrome de intestino irritable.
Se excluyeron pacientes con demencia tipo alzhéimer o demencia frontotemporal y aquellos que consumían medicamentos inductores de sueño, benzodiacepinas y antidepresivos.

Se definió como trastornos de sueño el resultado anormal en 1 o más de las escalas utilizadas.

\section{RESULTADOS}

Se incluyó un total de 80 pacientes con SII, la mayoría mujeres, con un promedio de edad de 35 años.

De acuerdo con los criterios y las escalas propuestas, en 38 pacientes $(47,5 \%)$ se encontró hipersomnia diurna por un valor mayor de 10 puntos en la escala de Epworth, 65 pacientes $(81 \%)$ mostraron algún grado de alteración en el índice de calidad de sueño medido con el cuestionario de Pittsburgh, 25 pacientes (31\%) presentaron puntajes compatibles con alteración moderada y severa; 41 pacientes (51\%) presentaron síndrome de piernas inquietas.

De la muestra total, 60 pacientes (75\%) presentaron alteración de la calidad de vida. Las características demográficas y clínicas de la muestra se encuentran en la tabla 1.

Tabla 1. Características demográficas y clínicas.

\begin{tabular}{ll}
\hline \multicolumn{1}{c}{ Características generales } & \multicolumn{1}{c}{ Total } \\
\hline Número de pacientes & 80 \\
Sexo masculino / femenino & $27,5 \%-72,5 \%$ \\
Edad promedio (años) & $35,5(\mathrm{DE} 12,94)$ \\
& $18-65$ años \\
& Hombres 39,9 (DE \\
& $14,73)$ \\
& Mujeres 33,8 (DE \\
& $11,91)$ \\
Epworth & \\
$<10$ puntos: normal & $52,50 \%(42)$ \\
Pittsburgh puntos: hipersomnia diurna & $47,50 \%(38)$ \\
5-7 puntos: merece atención médica & $18,75 \%(15)$ \\
8-13 puntos & $50 \%(40)$ \\
14- 21 puntos & $30 \%(24)$ \\
Alteración de la calidad vida SF-36 & $1,25 \%(1)$ \\
$<70$ puntos & \\
$>70$ puntos & $25 \%(20)$ \\
Piernas inquietas & $75 \%(60)$ \\
0 puntos &
\end{tabular}

Luego, al estratificar los desenlaces de acuerdo con la existencia de trastornos del sueño definidos por la altera- 
ción en 1 o más de las escalas usadas, y si existía algún grado de alteración de la calidad de vida según los resultados de la escala SF-36, se observó que la distribución por frecuencias en el grupo con trastorno del sueño se asoció con mayores alteraciones en la calidad de vida, con un OR de 4,8125, IC 95\%: 1,17-19,02, p <0,0076, diferencia que es estadísticamente significativa (tabla 2 ).

Tabla 2. Trastornos del sueño y alteración de la calidad de vida.

\begin{tabular}{lccc}
\hline & $\begin{array}{c}\text { Sin alteración } \\
\text { en la calidad } \\
\text { de vida }\end{array}$ & $\begin{array}{c}\text { Con alteración } \\
\text { en la calidad de } \\
\text { vida }\end{array}$ & Total \\
\hline $\begin{array}{l}\text { Con trastorno del sueño } \\
\text { (Pittsburgh }>4 \text { ) }\end{array}$ & 10 & 55 & 65 \\
Sin trastorno de sueño & 7 & 8 & 15 \\
\hline Total & 17 & 63 & 80 \\
\hline
\end{tabular}

OR 4,8125, IC 95\%: 1,17-19,02. $\mathrm{Chi}^{2}=7,13, \mathrm{p}<0,0076$

\section{DISCUSIÓN}

El síndrome de intestino irritable es una enfermedad muy frecuente dentro de la población general, alcanzando una prevalencia del $10 \%$ al $20 \%(9,10)$, y es uno de los principales motivos de consulta en todos los niveles de atención. Su morbilidad no depende solamente de la severidad de los síntomas digestivos, también son importantes las implicaciones sociales y culturales que llevan al paciente a limitar sus actividades diarias y a modificar su desempeño cotidiano, fenómeno que puede acentuarse con otras enfermedades con las cuales se puede acompañar, como fibromialgia y trastornos del sueño $(5,7,11)$.

Aunque se ha descrito una asociación entre SII y trastornos de sueño detectada con el estudio polisomnográfico (12), no hay información relevante en nuestro medio con la que se pueda establecer si las alteraciones de sueño que se manifiestan clínicamente (latencia prolongada del sueño, al igual que múltiples despertares nocturnos y somnolencia diurna) producen un mayor deterioro en la calidad de vida de los pacientes con SII (11).

Dentro de las herramientas existentes para valoración de los trastornos del sueño se encuentran la escala de somnolencia de Epworth, que evalúa la propensión a quedarse dormido en 8 situaciones sedentarias, así como el cuestionario de Berlín, que valora la presencia de ronquido, cese de respiración y somnolencia diurna; otro recurso diseñado para valorar el índice de calidad es el índice de sueño de Pittsburgh, al igual que el síndrome de piernas inquietas. Aunque el estudio polisomnográfico completo tiene mejores características operativas para detectar alteraciones de sueño, en el presente estudio se decidió utilizar escalas clínicas que fueran fácilmente reproducibles en cualquier ámbito clínico.

Los pacientes con SII usualmente reportan alteraciones en la calidad de vida comparables con las de otras enfermedades crónicas como diabetes, hipertensión o enfermedad renal crónica (1). Dentro de las escalas para valorar la calidad de vida se encuentran el cuestionario SF-36, así como IBS - QOL o IBS - HRQOL, dirigidos para pacientes con SII, al igual que la escala BEST, que puede permitir un acercamiento rápido a la calidad de vida de estos pacientes ( 1 , 8 ). Todas estas escalas tienen ventajas y desventajas en el momento de aplicarlas, y dada nuestra experiencia previa, la accesibilidad y la validación en español, y considerando que no hay grandes diferencias con las otras escalas descritas, se decidió usar la SF-36.

En este estudio se identificó que la prevalencia de trastornos del sueño en la población de pacientes con SII es alta (81\%), lo que podría ser explicado por el uso de varias escalas que evalúan diferentes desenlaces, con una buena sensibilidad para detectar alteraciones de sueño aunque con especificidad limitada. A pesar de ser instrumentos con relativamente baja especificidad, su ventaja radica en ser simples de aplicar e interpretar en cualquier escenario clínico.

La prevalencia de alteración en la calidad de vida según el SF-36 en estos pacientes con SII fue del 75\%, la cual se reconoce con una frecuencia alta y plausible dentro de la percepción de calidad de vida de este grupo de pacientes, que buscan atención médica y que usualmente cursan con cuadros moderados a severos.

Con los resultados obtenidos en este estudio se concluye que la asociación entre SII y trastornos de sueño, medida con escalas clínicas, es alta y se aproxima al $81 \%$. Existe una alta frecuencia $(75 \%)$ de alteración de la percepción de la calidad de vida (SF-36) en este grupo de pacientes con SII. También se encontró que la asociación entre SII y los trastornos de sueño se relaciona con una mayor alteración en la calidad de vida de los pacientes.

Este resultado debe alertar sobre la importancia de explorar alteraciones de sueño en los pacientes con SII y desarrollar estudios controlados para buscar si la detección y manejo de los trastornos de sueño en este tipo de pacientes puede producir resultados favorables en su calidad de vida.

\section{REFERENCIAS}

1. Agarwal N, Spiegel BM. The effect of irritable bowel syndrome on health-related quality of life and health care expenditures. Gastroenterol Clin North Am. 2011 Mar;40(1):11-19.

2. Videlock EJ, Chang L. Irritable bowel syndrome: Current approach to symptoms, evaluation, and treatment. Gastroenterol Clin North Am. 2007 Sep;36(3):665-85. 
3. Luscombe F. Health-related quality of life and associated psychosocial factors in irritable bowel syndrome: A review. Qual Life Res. 2000;9:161-176.

4. Poitras P, Gougeon A, et al. Extra digestive manifestations of irritable bowel syndrome: Intolerance to drugs. Dig Dis Sci. 2008 Aug;53(8):2168-76.

5. Riedl A, Schmidtmann M, et al. Somatic comorbidities of irritable bowel syndrome: A systematic analysis. J Psychosom Res. 2008 Jun;64(6):573-82. Epub 2008 Apr 28.

6. Amouretti M, Le Pen C, et al. Impact of irritable bowel syndrome (IBS) on health-related quality of life (HRQOL). Gastroenterol Clin Biol. 2006;30:241-246.

7. Orr WC. Sleep and gastrointestinal disease: A new horizon in sleep medicine. Sleep Med. 2009 Jun; 10(6):595-6.
8. Galdón Castillo A, Galera Mendoza L, et al. Disorders affecting the digestive system during sleep. Rev Neurol. 2004 Apr 16-30;38(8):757-65.

9. Spiller R, Aziz Q et al. Guidelines on the irritable bowel syndrome: Mechanisms and practical management. Gut. 2007;56:1770-1798.

10. Quigley EM, Bytzer P, et al. Irritable bowel syndrome: The burden and unmet needs in Europe. Dig Liver Dis. 2006 Oct;38(10):717-23.

11. Kanaly T, Shaheen NJ, et al. Gastrointestinal physiology and digestive disorders in sleep. Curr Opin Pulm Med. 15:571577.

12. Tibbits GM. Sleep Disorders: Causes, Effects, and Solutions. Prim Care. 2008 Dec;35(4):817-37. 